\title{
Optimising Ferrite-less Pad Reflection Winding with a Multi-Objective Genetic Algorithm
}

\author{
Matthew G. S. Pearce \\ Department of Electrical, \\ Computer and Software Engineering \\ University of Auckland \\ Auckland, New Zealand \\ matthew.pearce@auckland.ac.nz \\ Grant A. Covic \\ Department of Electrical, \\ Computer and Software Engineering \\ University of Auckland \\ Auckland, New Zealand \\ ga.covic@auckland.ac.nz
}

\author{
Michael J. O'Sullivan \\ Department of Engineering Science \\ University of Auckland \\ Auckland, New Zealand \\ michael.osullivan@auckland.ac.nz
}

\author{
Claudio Carretero \\ Department of Applied Physics \\ University of Zaragoza \\ Zaragoza, Spain \\ ccar@unizar.es
}

\author{
John T. Boys \\ Department of Electrical, \\ Computer and Software Engineering \\ University of Auckland \\ Auckland, New Zealand \\ j.boys@auckland.ac.nz
}

\begin{abstract}
Inductive power transfer is the leading technology for roadway electric vehicle wireless power transfer. A robust magnetic design is desired for magnetic couplers (pads) that are to be buried in a road surface. Ferrite-less designs including a reflection winding have been shown to be a possible way to increase the robustness of pads and reduce leakage magnetic field. This paper presents a methodology to optimise the reflection winding of a ferrite-less pad with a genetic algorithm and presents the results of an example optimisation with an axisymmetric approximation of the wireless power transfer standard SAE J2954 WPT3/Z3 secondary pad, with the results verified in the finite element analysis package FEMM, and in the laboratory. The results verify the reflection coil concept and indicate that improvements in leakage per power can be gained by allowing the reflection winding to be non-planar.
\end{abstract}

\section{INTRODUCTION}

Inductive Power Transfer (IPT) systems are becoming a commercial reality for Electric Vehicle (EV) wireless charging [1]. As transportation shifts to an electric future, robust wireless charging infrastructure will become commonplace. Roadway IPT magnetic couplers (pads) present challenges different to other applications of IPT, in that they need to be able to transfer a high power, with a low leakage magnetic field where a person may be present. Pads also need to have a long service life in a harsh environment. While the brittle magnetic material ferrite improves the coupling between pads, it's fragile nature does not belong in a roadway environment. Recent research into ferrite-less and reduced ferrite systems has showed promising results [2]-[4]. This paper focuses on the techniques for the optimisation of axisymmetric ferriteless roadway magnetic couplers with reflection coils with the use of a genetic algorithm (GA). Optimisation algorithms have been used before to optimise IPT pads [5]-[9], but not for the design of reflection windings.
Improvements in the performance of the simple ferriteless IPT system have been proposed based on the insertion of an additional coil in the primary side to reduce the total leakage. Among others, one of the most promising structures is series-connecting additional turns in opposing-sense to the primary, that is, carrying a current $-I_{1}$. In that configuration, the original concentric turn primary winding are called a driven winding and the additional turns are called a reflection winding [3]. In early proposals, this reflection winding is composed of several turns coplanar with or, alternatively, placed underneath the driven winding. The objective is a reduced magnetic field leakage and self inductance for a minimal impact on $M_{12}$, (the mutual coupling between the primary pad and the secondary pad on a vehicle).

\section{A. Paper overview}

The objective of this work is finding an optimal non-planar reflection winding using a GA. The metrics are a reduced side and bottom magnetic field leakage and primary VA, $\mathrm{VA}_{1}$ for a set power transferred to the vehicle. A general background to roadway IPT systems is provided in Sec. II, including a description of the figure of merit used in this paper. Sec. III presents the analytic mathematical techniques to model this system. The multi-objective GA is then presented in Sec. IV. Then the GA is applied with different constraints to the magnetic system and the results discussed in Sec. V, and a verification of the analytical techniques against finite element analysis simulations and laboratory work is presented in Sec VI. Finally Sec. VII presents recommendations for optimisation of such magnetic systems and Sec. VIII concludes. 


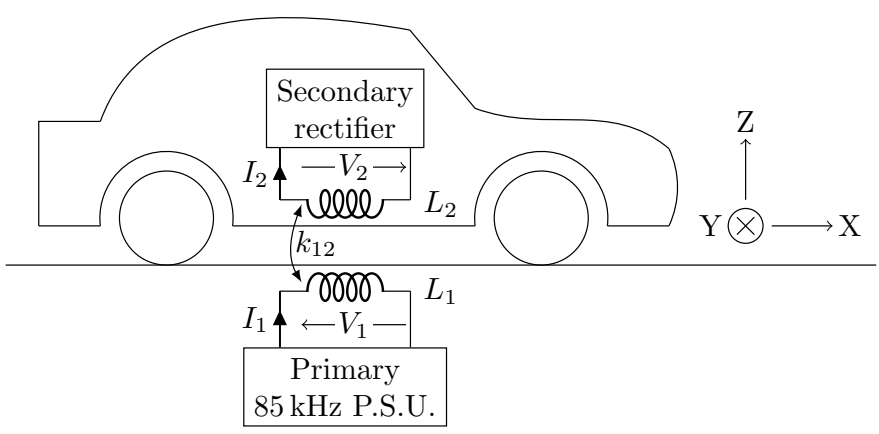

Fig. 1. High level overview of an EV IPT system.

\section{BACKGROUND TO IPT SYSTEMS}

Fig. 1 depicts a high level overview of an IPT system. Such IPT systems transfer power with the aid of a magnetic field between two loosely coupled inductors. In an IPT system, the power transferred is proportional to the secondary loaded quality factor of the tuned circuit [10], $P_{\mathrm{OUT}}=S_{\mathrm{U}} Q_{2}$, where $P_{\text {OUT }}$ is the output power, and the $S_{\mathrm{U}}$ is derived from the product of the open circuit voltage $\left(V_{\mathrm{OC}}=j \omega M_{12} I_{1}\right)$ of the secondary pad and the short circuit current $\left(I_{\mathrm{SC}}=\frac{V_{\mathrm{OC}}}{j \omega L_{2}}\right)$ to find the uncompensated secondary apparent power,

$$
S_{\mathrm{U}}=\omega\left(\frac{M_{12}^{2}}{L_{2}}\right) I_{1}^{2}=\omega\left(k_{12}^{2} L_{1}\right) I_{1}^{2},
$$

where $\omega=2 \pi f$ is the operating angular frequency, $M_{12}$ is the mutual inductance between the two coupled inductors, $k_{12}$ is the coupling factor $\left(k_{12}=\frac{M_{12}}{\sqrt{L_{1} L_{2}}}\right), L_{1}$ and $L_{2}$ are the self inductances and $I_{1}$ is the current in the primary.

To simplify the analysis the secondary loaded quality factor $Q_{2}$ is neglected for this analysis [10]. This allows (1) to be used as the figure of merit for power delivery.

There are 3 key design metrics in this work, the primary VA required to delivered the desired output apparent power $S_{\mathrm{U}_{d}}$, and the maximum leakage magnetic field $\left(B_{\mathrm{RMS}}\right)$ observed in the radial direction (side leakage) and below the top surface of the primary (bottom leakage).

The primary VA required to deliver the desired $S_{\mathrm{U}}$, is called $\mathrm{VA}_{1}$ and may be calculated from (1), $\mathrm{VA}_{1}=\frac{S_{\mathrm{U}_{d}}}{k_{12}^{2}}$.

The side leakage magnetic field is taken as the maximum field observed at a distance of $800 \mathrm{~mm}$ from the centre of the secondary. This represents the side of a vehicle - a car being around $1.6 \mathrm{~m}$ wide — but any set of points could be chosen in future analyses. The bottom leakage field is taken as $300 \mathrm{~mm}$ below the top surface of the pad (the road surface) and is chosen as a plausible distance down to the road bed. These are illustrated in Fig. 2.

Power transfer capabilities are limited by the maximum leakage flux established by the regulations. The secondary pad is short-circuited to estimate a parallel tuned secondary [10] meaning $I_{2}=I_{\mathrm{SC}}=-\frac{M_{12}}{L_{2}} I_{1}$ and $I_{1}$ is calculated from (1) for $S_{\mathrm{U}}=1 \mathrm{kVA}$. Note the secondary's current is out of phase and proportional to the primary current,

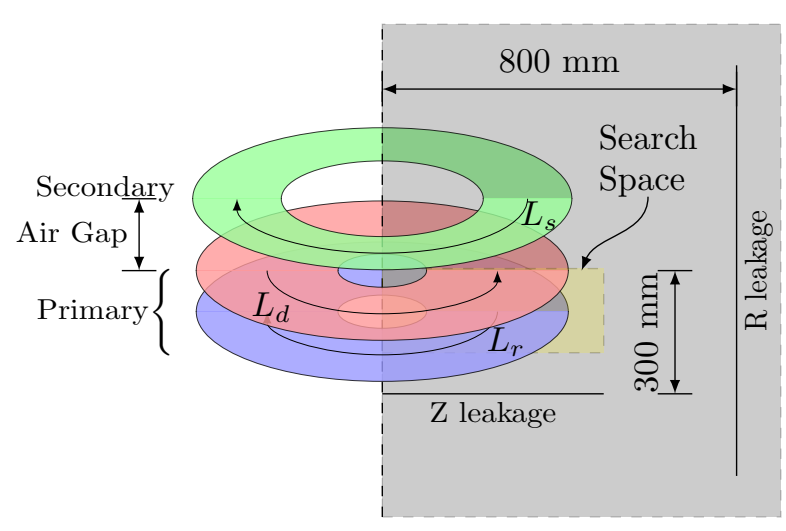

Fig. 2. Primary pad made up of driven and reflection winding, and secondary pad. Also shown are the leakage measurement lines to the side and below pad.

simplifying the description of the leakage in the system to, $\mathbf{B}_{\mathrm{IPT}}\left(\mathbf{r}^{\prime}\right)=\mathbf{b}_{1}\left(\mathbf{r}^{\prime}\right) I_{1}+\mathbf{b}_{2}\left(\mathbf{r}^{\prime}\right) I_{\mathrm{SC}}$, where $\mathbf{b}_{1}\left(\mathbf{r}^{\prime}\right)$ and $\mathbf{b}_{2}\left(\mathbf{r}^{\prime}\right)$ are the normalised primary and secondary leakage.

The leakage and $\mathrm{VA}_{1}$ must be calculated with each pad delivering the same power to the secondary. This simultaneously takes into account the variations in coupling and self inductance and magnetic field pattern that a certain primary geometry possesses when coupled to the same secondary.

\section{Magnetics}

A simplified axi-symmetric ferrite-less IPT system is chosen to provide a platform to explore the issues of applying optimisation algorithms to IPT magnetic couplers. An conceptual representation of this is shown in Fig. 2. Because this is an axi-symmetric system, every element (loop) can be represented as a $(r, z)$ coordinate pair where $r$ is the radius and $z$ is the height of the loop.

This initial IPT system consists of two planar coils of $N_{1}$ and $N_{2}$ concentric turns for the primary and secondary pads, respectively. The air gap between the primary and secondary pads corresponds to their axial distance.

The self inductance of the primary and secondary were evaluated numerically using the equation, $L=\sum_{i=1}^{N} L_{i i}+$ $\sum_{i=1}^{N} \sum_{j=1, j \neq i}^{N} M_{i j}$, and the mutual inductance between the primary and secondary with, $M_{12}=\sum_{i=1}^{N_{1}} \sum_{j=1}^{N_{2}} M_{i j}$, where [11], [12],

$$
L_{i i}=\mu_{0} r_{i}\left(\ln \left(\frac{16 r_{i}}{d_{w}}\right)-1.75\right),
$$

and

$$
M_{i j}=\mu_{0} \frac{\sqrt{r_{i} r_{j}}}{k_{i j}}\left(\left(2-k_{i j}^{2}\right) \mathrm{K}\left(k_{i j}^{2}\right)-2 \mathrm{E}\left(k_{i j}^{2}\right)\right),
$$

$\mu_{0}=4 \pi \times 10^{-7} \mathrm{H} / \mathrm{m}$ is the permeability of free space, $d_{w}$ is the diameter of the wire, $\left(r_{i}, z_{i}\right)$ are the coordinates of the first loop, $\left(r_{j}, z_{j}\right)$ are the coordinates of the second loop, and $\mathrm{K}\left(k_{i j}^{2}\right)$ and $\mathrm{E}\left(k_{i j}^{2}\right)$ are the complete elliptic integrals of first and second kinds with, 


$$
k_{i j}=\sqrt{\frac{4 r_{i} r_{j}}{\left(r_{i}+r_{j}\right)^{2}+\left(z_{j}-z_{i}\right)^{2}}} .
$$

The magnetic field's components of such loop (radial and axial components) are provided by the following expressions [13]:

$$
\begin{aligned}
& B_{r}\left(r_{i}, r_{j}, z_{j}-z_{i}\right) \\
& =\mu_{0} I_{1}\left(z_{j}-z_{i}\right) k_{i j} \times \frac{\left(2-k_{i j}^{2}\right) \mathrm{E}\left(k_{i j}^{2}\right)-2\left(1-k_{i j}^{2}\right) \mathrm{K}\left(k_{i j}^{2}\right)}{8 \pi\left(1-k_{i j}^{2}\right)\left(r_{i} r_{j}^{3}\right)^{1 / 2}}
\end{aligned}
$$

and

$$
\begin{aligned}
& B_{z}\left(r_{i}, r_{j}, z_{j}-z_{i}\right)=\mu_{0} I_{1} k_{i j} \times \\
& \frac{k_{i j}^{2}\left(r_{i}^{2}-r_{j}^{2}-\left(z_{j}-z_{i}\right)^{2}\right) \mathrm{E}\left(k_{i j}^{2}\right)+4 r_{i} r_{j}\left(1-k_{i j}^{2}\right) \mathrm{K}\left(k_{i j}^{2}\right)}{16 \pi\left(1-k_{i j}^{2}\right)\left(r_{i} r_{j}\right)^{3 / 2}}
\end{aligned}
$$

where $r_{i}, z_{j}$ are the coordinates representing the loop, $r_{j}, z_{j}$ are the coordinates representing the measurement point.

\section{DESIGNING IPT PADS USING A Multi-Objective Genetic Algorithm}

GAs are a class of metaheuristic inspired by both the way genetic material in nature evolves and also how this genetic material informs the "fitness" of an individual and, hence, its ability to survive. GAs (and other metaheuristics) are frequently applied to black-box optimisation problems. IPT pad design can be modelled as a black-box optimisation problem given that the mathematical models for power and magnetics (see Sec. II and Sec. III) define how the $(r, z)$ positions of the loops affect both power output and the magnetic field produced by the pad.

Traditionally, black-box optimisation deals with a single output value that is optimised (usually minimised), known as the objective. However, in the IPT design problem (and other design problems), multiple competing output value are considered and trade-offs between these values inform designs that are "efficient", i.e., in which the value of one output cannot be improved without degrading one of the other output values. Finding a set of these efficient designs can be achieved by solving a a multi-objective black-box optimization problem. NSGA-II is a multi-objective GA [14] that has been implemented as a Python library [15] based on the DEAP evolutionary framework [16]. In this paper the Python implementation of NSGA-II is used to find a set of pad designs that, while not guaranteed to be efficient, will be close to being efficient (since GAs are metaheuristics with no guarantee of optimality).

In order to identify the best designs from the set of designs produced by the NSGA-II Python implementation, a technique called Data Envelopment Analysis (DEA) is used. DEA is a technique that can be used to benchmark the performance of entities (usually manufacturing or service operations) that are measured using multiple metrics. DEA can identify the efficient entities within a set of entities, i.e., entities with metrics values that cannot all be bettered by another entity. In this paper an open source implementation of DEA, pyDEA [17], is used to post-process the set of pad designs produced by the multi-objective GA to identify the efficient designs. These efficient designs are then manually filtered to produce the designs presented Sec. V.

The remainder of this section is structured as follows. The pad design problem is summarised in Sec. IV-A and the parameters of the NSGA-II implementation are then described in Sec. IV-B. Finally, the use of DEA to determine the best pad designs is outlined in Sec. IV-C.

\section{A. IPT Pad Design Problem}

The IPT pad design problem is relatively simple. The design parameters are the $(r, z)$ values and current sense for each loop and bounds on these values are supplied to the GA. The GA then generates and evolves a set of designs that define the loops and the equations in Sec. II and III produce the outputs values, namely bottom leakage, side leakage, and $\mathrm{VA}_{1}$.

As the GA progresses, the designs produced will be close to being efficient in all three outputs, i.e., none of the outputs can be decreased without increasing at least one of the others. In some experiments thresholds on the outputs from previous known good designs were used to direct the designs to produce output values better than the best currently known.

\section{B. Multi-Objective Genetic Algorithm}

The parameters of Reszelewski's Python implementation of NSGA-II that were used are given below, with an indication of whether this was a default parameter value. Note that understanding these parameters requires some knowledge of GAs, the interested reader is directed to Introduction and Core Architecture sections of [16] and the references contained in those sections.

- All objective weightings are set at -1 so the GA will try to minimize all the outputs and consider them equally;

- Designs will be combined, e.g., a mix of loops from two designs are be used to create a new design, using a simulated binary crossover with a "crowding degree of the crossover" $\eta=20.0$ (default) - see [16] for details;

- Perturbing designs to ensure good coverage of the possible design space is performed using polynomial mutation (as implemented in original NSGA-II algorithm in C by Deb [14]) with crowding degree $\eta=20.0$ and the independent probability for each design variable set to $\frac{1}{n}$ where $n=$ the number of design variables, i.e., each variable is equally likely to be mutated (default);

- Selecting designs to remain in the design set, i.e., keeping designs that are close to being efficient, is performed using the NSGA-II selection operator, see [14] for details (default).

\section{Identifying Efficient Designs using Data Envelopment Analysis}

The set of designs produced by the NSGA-II Python implementation consists of a set of loop $(r, z)$ values and current 


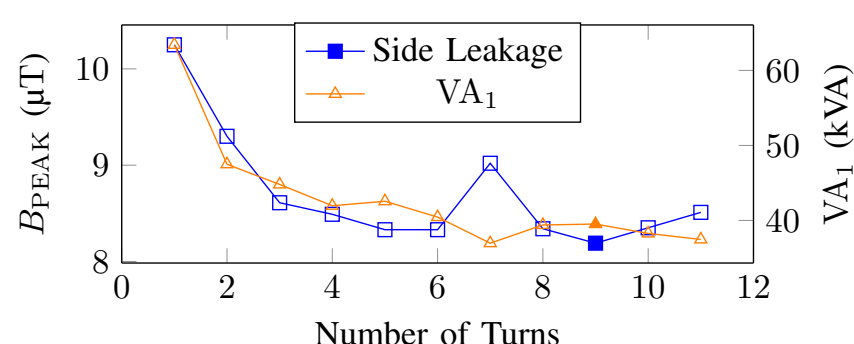

Fig. 3. Leakage and $\mathrm{VA}_{1}$ while delivering $S_{\mathrm{U}}=1 \mathrm{kVA}$ as the number of turns in a driven winding changes.

sense and the corresponding output values for bottom leakage, side leakage, and $\mathrm{VA}_{1}$, produced from the equations in Sec. II and III.

DEA looks for entities that use minimal inputs to create maximal outputs, i.e., that are efficient in converting inputs to outputs. The smallest GA output values that result in $1 \mathrm{kVA}$ of $S_{\mathrm{U}}$ are desired so the GA output values of each design are set as the necessary inputs in pyDEA to produce an output $S_{\mathrm{U}}$ of $1 \mathrm{kVA}$. The pyDEA software is then run with all other settings as defaults and this produces a list of the designs and their efficiency (between 0 and 1) within the set of designs. All designs with an efficiency of 1 are efficient within the set of designs and are selected for further consideration.

\section{Application to SAE J2954 WPT3 Secondary}

The secondary pad is modelled as a ferrite-less axisymmetric approximation of the SAE J2954 WPT3/Z3 secondary pad [18] and consists of 8 evenly spaced circular turns, with an inner radius of $114 \mathrm{~mm}$ and an outer radius of $214 \mathrm{~mm}$, made up of $4 \mathrm{~mm}$ diameter litz wire. It is at an air gap of $175 \mathrm{~mm}$ above the primary pad, and has a calculated self inductance of $26.8 \mu \mathrm{H}$. The centre of the primary coil is defined as $(0,0)$, and the search space is discretised into $5 \mathrm{~mm}$ steps, as the litz wires used are $4 \mathrm{~mm}$ in diameter. All the reported results are with the secondary receiving an $S_{\mathrm{U}}=1 \mathrm{kVA}$.

\section{A. Optimising the Driven Winding}

The GA was run 11 times with 1-11 free elements, 300 generations of 100 individuals with the design elements (primary turns) constrained to $Z=0$ (top of primary), and any radius from $r=0$ to $r=500 \mathrm{~mm}$. From the population, the individual with lower side leakage at $800 \mathrm{~mm}$ and/or $\mathrm{VA}_{1}$ than the previous number of turns is selected. A low side leakage is focused on here, but individuals with the lowest bottom leakage could be selected in another analysis. Due to the nature of the GA both the bottom and side leakage are low. Results are shown in Fig. 3. A driven winding of 9 loops is selected as it has the lowest side leakage, for a small $\mathrm{VA}_{1}$.

The 9 loop individual was then hand optimised to make it more regular with the results presented in Tab. I. The loop radii are $[0.05,0.07,0.09,0.11,0.13,0.15,0.17,0.19,0.21] \mathrm{m}$, and are included in Tab. II. This winding is then used as the basis of the fixed- $z$ and free- $z$ evaluations.

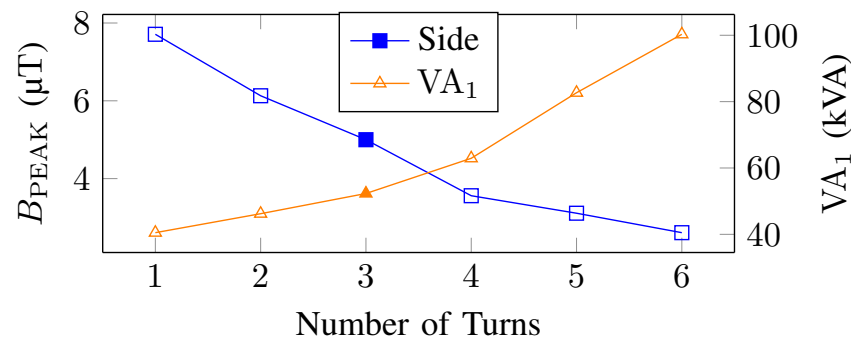

Fig. 4. Leakage and $\mathrm{VA}_{1}$ while delivering $S_{\mathrm{U}}=1 \mathrm{kVA}$ as the number of turns of a planar reflection winding (fixed- $z$ ) changes, with a fixed driven winding.

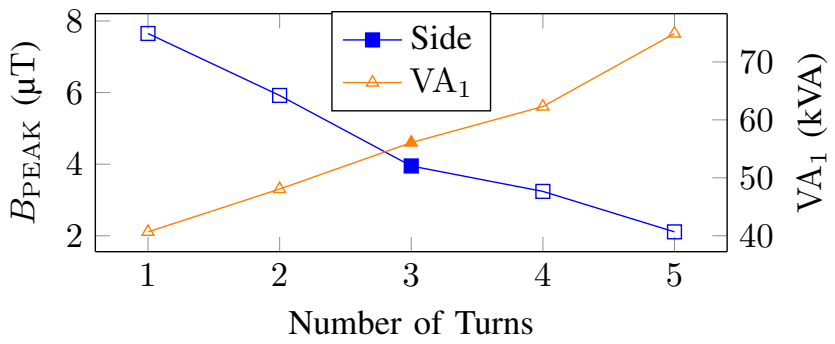

Fig. 5. Leakage and $\mathrm{VA}_{1}$ while delivering $S_{\mathrm{U}}=1 \mathrm{kVA}$ as the number of turns of a freely placed reflection winding (free- $z$ ) changes, with a fixed driven winding.

\section{B. Reflection Winding - Fixed-z}

To further decrease the leakage a directly driven reflection winding is added [3], as illustrated in Fig. 2. This consists of turns with a current in an opposite sense to the driven winding, and will increase the $\mathrm{VA}_{1}$ needed to deliver power. In this case, the optimiser is constrained so that it uses the driven winding found in the previous section. In addition, the reflection winding is constrained such that it has all the loops on an GA chosen $z$ plane (fixed- $z$ ) below the main driven winding, and the loops all have a radius larger than the driven winding inner radius. The total search space for the primary consists of an area from $5 \mathrm{~mm}$ to $500 \mathrm{~mm}$ in the radial direction and from 0 to $-200 \mathrm{~mm}$ in the axial direction, shown with a shaded box in Figs. 2 and 6.

The optimiser was run 6 times changing the number of loops

TABLE I

METRICS OF SELECTED INDIVIDUALS, INCLUDING VERIFICATION OF THE ALL-FREE OPTIMISATION.

\begin{tabular}{cccccc}
\hline & $\begin{array}{c}\text { Side } \\
800 \mathrm{~mm} \\
(\mathrm{uT} \text { peak })\end{array}$ & $\begin{array}{c}\text { Bottom } \\
300 \mathrm{~mm} \\
(\mathrm{uT} \text { peak })\end{array}$ & $\mathrm{VA}_{1}$ & $L_{1}$ & $k$ \\
$(\mathrm{kVA})$ & $(\mu \mathrm{H})$ & $(-)$ \\
\hline \hline Driven & 8.44 & 222.10 & 37.36 & 19.20 & 0.1636 \\
Fixed- $z$ & 5.00 & 31.73 & 52.31 & 19.10 & 0.1383 \\
Free- $z$ & 3.95 & 17.02 & 56.08 & 19.49 & 0.1334 \\
All-Free & 3.29 & 9.82 & 49.48 & 23.49 & 0.1422 \\
\hline AF Code & 3.29 & 9.82 & 49.48 & 23.49 & 0.1422 \\
AF FEMM & 3.29 & 9.76 & 49.00 & 23.32 & 0.1429 \\
AF Lab & 3.48 & 11.80 & 49.78 & 24.15 & 0.1335 \\
\hline
\end{tabular}




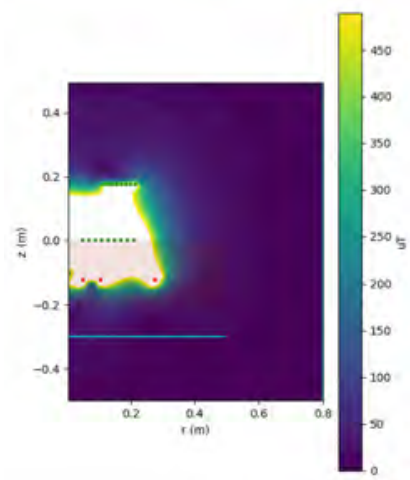

(a) Fixed- $z$

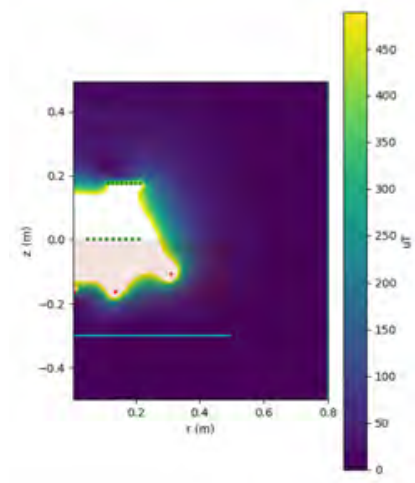

(b) Free- $z$

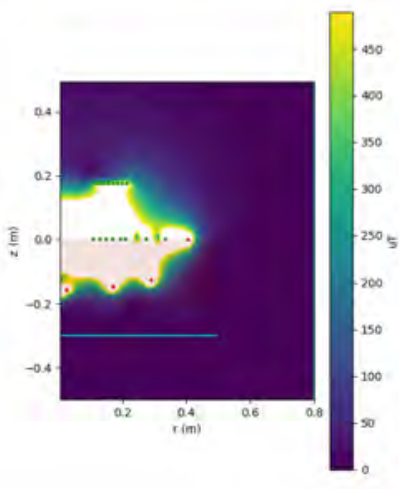

(c) All Free

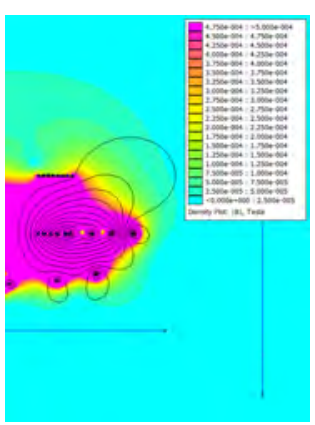

(d) All Free FEMM

Fig. 6. Selected individuals from (a) fixed- $z$ (Sec. V-B), (b) free- $z$ (Sec. V-C), (c) All Free (Sec. V-D) and (d) FEMM verification of All Free. See Tab. I for metric values.

from 1-6 [3]. Fig. 4 show a selected individual for each of the 6 runs. These were chosen to always reduce the maximum side leakage with minimal increase in $\mathrm{VA}_{1}$.

Fig. 4 shows how there is a trade-off between decreasing side leakage and increasing $\mathrm{VA}_{1}$. In this instance, 3 turns are selected as an example, as a good tradeoff between a decrease in side leakage (42\%) and increase in $\mathrm{VA}_{1}(39 \%)$ from the no reflection winding option. The geometry is shown in Fig. 6 a.

\section{Reflection Winding - Free- $z$}

Next, the optimisation algorithm was run again. The driven winding was kept the same as before, but now the reflection winding turns are allowed to be placed at any $r$ or $z$ coordinate inside the search space. The algorithm was run 6 times with up to 6 loops.

Fig. 5 shows the results, with the selected individual in Tab I and the geometry in Fig. 6b. Note the non-planar bowl shape and a reduction in side leakage and a minimal increase in $\mathrm{VA}_{1}$ from the planar case. The coordinates of the specific loops are provided in Tab. II.

\section{All-Free}

Finally the optimiser was run with 12 elements able to be placed anywhere. A selected individual is shown in Fig. 6c. As can be seen this individual consists of an 8 turn driven winding and a 4 turn reflection winding, where the reflection winding is in a bowl shape.

This pad, being one of the best performing is chosen for Finite Element Analysis (FEA) and laboratory verification, covered in the next section.

\section{LABORATORY VERIFICATION}

The all-free optimisation discussed above and shown in Fig. 6c was verified with the freely available FEA magnetics package FEMM [19], with the program output shown in Fig. 6d, and the metrics provided in Tab. I. This same allfree optimisation was constructed and run in the laboratory, with the results also given in Tab. I.

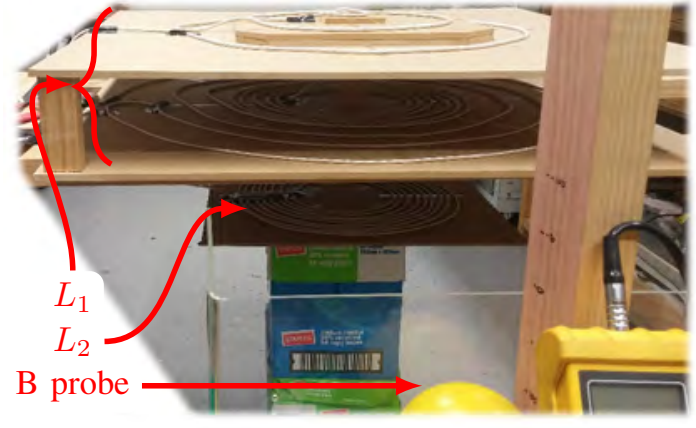

Fig. 7. Laboratory setup, showing primary (top) and secondary (bottom) pads, with the flux measurement proble located $800 \mathrm{~mm}$ from the centre of the system.

The self inductances and coupling were measured with an Agilent E4980A LCR meter. Then the pad was parallel compensated with a $148 \mathrm{nF}$ capacitor and run, with the secondary shorted as in simulation, with a linear amplifier at the tank circuit's resonant frequency $(83.88 \mathrm{kHz})$ to minimise the reactive load on the linear amplifier. The leakage was measured with a Narda ELT-400 flux measurement probe. A photograph of the laboratory setup is shown in Fig. 7; note the prototype was constructed and photographed upside down to emphasise the reflection winding.

Tab. I shows good agreement between the optimisation algorithm, FEMM and laboratory verification of the all-free optimisation. Small discrepancies are from meshing errors in simulation, with long leads and spatial inaccuracies causing the slight differences in the laboratory results. The leakage reported is at the coordinate of the measured maximum in simulation. The bottom leakage laboratory results had a higher maximum due to the extra wires needed to connect the loops.

\section{DISCUSSION}

This paper has presented a technique, demonstrated a tool and added understanding to the design of a reflection winding, which up until this point has been planar. With a specific 
TABLE II

COORDINATES OF ALL LOOPS OF SELECTED INDIVIDUALS

\begin{tabular}{|c|c|c|c|c|c|c|c|c|c|c|c|}
\hline \multicolumn{3}{|c|}{ Secondary } & \multicolumn{3}{|c|}{ Fixed-z } & \multicolumn{3}{|c|}{ Free-z } & \multicolumn{3}{|c|}{ All Free } \\
\hline $\begin{array}{c}\mathrm{r} \\
(\mathrm{mm})\end{array}$ & $\begin{array}{c}\mathrm{Z} \\
(\mathrm{mm})\end{array}$ & $\begin{array}{l}\mathrm{i} \\
-\end{array}$ & $\begin{array}{c}\mathrm{r} \\
(\mathrm{mm})\end{array}$ & $\begin{array}{c}\mathrm{Z} \\
(\mathrm{mm})\end{array}$ & $\begin{array}{l}\mathrm{i} \\
-\end{array}$ & $\begin{array}{c}\mathrm{r} \\
(\mathrm{mm})\end{array}$ & $\begin{array}{c}\mathrm{Z} \\
(\mathrm{mm})\end{array}$ & $\begin{array}{l}\mathrm{i} \\
-\end{array}$ & $\begin{array}{c}\mathrm{r} \\
(\mathrm{mm})\end{array}$ & $\begin{array}{c}\mathrm{Z} \\
(\mathrm{mm})\end{array}$ & $\begin{array}{l}\mathrm{i} \\
-\end{array}$ \\
\hline 114 & 175 & 1 & 50 & 0 & 1 & 50 & 0 & 1 & 110 & 0 & 1 \\
\hline 129 & 175 & 1 & 70 & 0 & 1 & 70 & 0 & 1 & 130 & 0 & 1 \\
\hline 143 & 175 & 1 & 90 & 0 & 1 & 90 & 0 & 1 & 150 & 0 & 1 \\
\hline 157 & 175 & 1 & 110 & 0 & 1 & 110 & 0 & 1 & 170 & 0 & 1 \\
\hline 172 & 175 & 1 & 130 & 0 & 1 & 130 & 0 & 1 & 195 & 0 & 1 \\
\hline 186 & 175 & 1 & 150 & 0 & 1 & 150 & 0 & 1 & 210 & 0 & 1 \\
\hline 200 & 175 & 1 & 170 & 0 & 1 & 170 & 0 & 1 & 275 & 0 & 1 \\
\hline 214 & 175 & 1 & 190 & 0 & 1 & 190 & 0 & 1 & 335 & 0 & 1 \\
\hline & & & 210 & 0 & 1 & 210 & 0 & 1 & 25 & -155 & -1 \\
\hline & & & 50 & -120 & -1 & 5 & -160 & -1 & 170 & -145 & -1 \\
\hline & & & 105 & -120 & -1 & 135 & -160 & -1 & 290 & -125 & -1 \\
\hline & & & 275 & -120 & -1 & 310 & -105 & -1 & 405 & 0 & -1 \\
\hline
\end{tabular}

application in mind this method can be applied and, by constraining the solver as described, an optimal solution can be reached very quickly. Note that the selection of individuals is highly dependant on the final application and is hard to automate.

In this simplified axisymmetric ferrite-less example, the all-free optimisation produced a result similar to the free- $z$ reflection winding (with many loops at the uppermost edge of the search space and the reflection winding in a bowl shape). The optimal fixed- $z$ reflection winding and the free- $z$ reflection winding show that a more bowl shape is desirable, as seen in Tab. I and Fig. 6. While the optimisation of the driven winding on its own produced a good result, the final all-free optimisation produced an 8 turn driven winding and a 4 turn reflection winding utilising a co-planar anti-phase loop to cancel the leakage to the side.

These results highlight how different constraints on the GA can produce different optimal practical solutions. For instance the fixed- $z$ reflection winding is simpler to manufacture than the free- $z$ reflection winding, and the all-free result is the most complex to construct, but produces a good magnetic result.

\section{CONCLUSION}

Given the large size of the search space for ferrite-less pads, a GA allows an expedient discovery of an optimal geometry. The free- $z$ reflection winding produced a higher performing system than without a reflection winding, and in addition the all-free optimisation produces an even better result and verifies the reflection coil concept. The methodology used in this paper can be used to quickly find an optimal pad for a given set of leakage and space requirements.

\section{REFERENCES}

[1] WiTricity, "WiTricity automotive." Available at http://witricity.com/ technology/automotive/, accessed $8^{\text {th }}$ April 2018.

[2] M. G. S. Pearce, G. A. Covic, and J. T. Boys, "Robust ferrite-less Double D topology for roadway IPT applications," IEEE Transactions on Power Electronics, p. 1, 2018.

[3] A. Tejeda, C. Carretero, J. T. Boys, and G. A. Covic, "Ferrite-less circular pad with controlled flux cancelation for EV wireless charging," IEEE Transactions on Power Electronics, vol. 32, pp. 8349-8359, Nov. 2017.
[4] S. Y. Choi, B. W. Gu, S. Y. Jeong, and C. T. Rim, "Advances in wireless power transfer systems for roadway-powered electric vehicles," IEEE Journal of Emerging and Selected Topics in Power Electronics, vol. 3, pp. 18-36, Mar. 2015.

[5] Z. Luo, X. Wei, and G. A. Covic, "Multi-objective optimization of double D coils for wireless charging system," in Proc. IEEE Int. Power Electronics and Application Conf. and Exposition (PEAC), pp. 1-6, Nov. 2018.

[6] S. Bandyopadhyay, V. Prasanth, P. Bauer, and J. A. Ferreira, "Multiobjective optimisation of a $1-\mathrm{kW}$ wireless IPT systems for charging of electric vehicles," in 2016 IEEE Transportation Electrification Conference and Expo (ITEC), pp. 1-7, June 2016.

[7] R. Bosshard and J. W. Kolar, "Multi-objective optimization of $50 \mathrm{~kW} / 85 \mathrm{kHz}$ IPT system for public transport," IEEE Journal of Emerging and Selected Topics in Power Electronics, vol. 4, pp. 13701382, Dec. 2016.

[8] R. Bosshard, J. W. Kolar, J. Mühlethaler, I. Stevanović, B. Wunsch, and F. Canales, "Modeling and $\eta$ - $\alpha$-pareto optimization of inductive power transfer coils for electric vehicles," IEEE Journal of Emerging and Selected Topics in Power Electronics, vol. 3, pp. 50-64, Mar. 2015.

[9] T. Yilmaz, N. Hasan, R. Zane, and Z. Pantic, "Multi-objective optimization of circular magnetic couplers for wireless power transfer applications," IEEE Transactions on Magnetics, vol. 53, pp. 1-12, Aug. 2017. Art no. 8700312.

[10] J. T. Boys, G. A. Covic, and A. W. Green, "Stability and control of inductively coupled power transfer systems," IEE Proceedings-Electric Power Applications, vol. 147, pp. 37-43, Jan. 2000.

[11] J. A. Stratton, Electromagnetic theory. McGraw-Hill New York, 1st ed. ed., 1941.

[12] J. Clerk Maxwell, A Treatise on Electricity and Magnetism. Walton Street, Oxford OX2 6DP, UK: Oxford University Press, 1871.

[13] J. T. Conway, "Analytical and semi-analytical solutions for the force between circular loops in parallel planes," IEEE Transactions on Magnetics, vol. 49, pp. 4817-4823, Aug. 2013.

[14] K. Deb, A. Pratap, S. Agarwal, and T. Meyarivan, "A fast and elitist multiobjective genetic algorithm: NSGA-II," IEEE Transactions on Evolutionary Computation, vol. 6, pp. 182-197, April 2002.

[15] W. Reszelewski, "Implementation of NSGA-II algorithm in form of a python library." https://github.com/wreszelewski, date $=2014$.

[16] F.-A. Fortin, F.-M. De Rainville, M.-A. Gardner, M. Parizeau, and C. Gagné, "DEAP: Evolutionary algorithms made easy," Journal of Machine Learning Research, vol. 13, pp. 2171-2175, jul 2012.

[17] R. A., H. K., L. A., P. H., and R. M, "pyDEA - a software package and user interface for DEA," tech. rep., Department of Engineering Science, The University of Auckland, 2012.

[18] SAE International, "Wireless Power Transfer for Light-Duty Plug-In/ Electric Vehicles and Alignment Methodology," J2954, Nov. 2017.

[19] D. C. Meeker, "Finite Element Method Magnetics, Version 4.2 (28Feb2018 Build)." 